\title{
Targeted Enhancement of the Biological Activity of the Antineoplastic Agent, Neocarzinostatin

\author{
Studies in Murine Neuroblastoma Cells
}

\author{
Nina Felice Schor \\ Departments of Pediatrics, Neurology, and Pharmacology, University of Pittsburgh School of Medicine, Pittsburgh, Pennsylvania 15213
}

\begin{abstract}
The development of chemotherapeutic approaches to cancer has been hampered by the toxicity of proposed agents for normal rapidly dividing cells. By using neocarzinostatin, a "prodrug" which is activated by reduction by thiol compounds, adjunctively with 6-mercaptodopamine, a thiol-containing dopamine analogue, we have been able to enhance neocarzinostatin toxicity for cells of the neural crest tumor neuroblastoma. Thiol compounds that are not neurotransmitter analogues do not act synergistically with neocarzinostatin in this system. Since most normal rapidly dividing cells do not have surface dopamine receptors, we propose this approach for the selective targeting of toxicity for neuroblastoma cells. We further introduce cell-selective activation of prodrugs as a new chemotherapeutic strategy which demands further development. (J. Clin. Invest. 1992. 89:774-781.) Key words: differentiation inducers $\bullet$ neural crest tumors • dopamine
\end{abstract}

\section{Introduction}

The designers of cancer chemotherapy are charged with the seemingly paradoxical duty of designing agents which are, on the one hand, cytocidal for tumor cells, and, on the other hand, not overwhelmingly toxic to normal cells. Since most chemotherapeutic strategies take advantage of quantitative, rather than qualitative, differences between normal and neoplastic cells, many chemotherapeutic agents are extremely toxic to normal tissues. This is especially true for normal tissues with high mitotic rates (1). Studies in our laboratory aimed at designing new chemotherapeutic strategies for neuroblastoma, a neural crest tumor, take specific advantage of the neural characteristics of such cells. Using a "prodrug", which must be activated to be toxic, along with novel activating agents which are chemical analogues of neurotransmitters, we can specifically enhance prodrug activation (and thereby increase cytotoxicity) in neural crest cells.

Neocarzinostatin is an antimitotic natural product consisting of a chromophore and a protein of $10 \mathrm{kD}$ molecular mass to which it is noncovalently bound (2). We have previously shown that neocarzinostatin is antimitotic for murine neuro-

Address correspondence to Dr. Nina Felice Schor, Division of Child Neurology, Children's Hospital of Pittsburgh, 3705 Fifth Avenue, Pittsburgh, PA 15213.

Received for publication 23 July 1991 and in revised form 29 October 1991.

J. Clin. Invest.

(c) The American Society for Clinical Investigation, Inc.

0021-9738/92/03/0774/08 \$2.00

Volume 89, March 1992, 774-781 blastoma cells, and that it induces these cells to adopt the morphology of neurons (3). Others have shown that the antimitotic activity of neocarzinostatin is dependent upon the reduction of its chromophore by sulfhydryl groups. Both in vitro and in vivo studies have shown the relationship between sulfhydryl concentration and the cytostatic potency of neocarzinostatin $(4,5)$. We have used the approach of enriching the sulfhydryl content of dopamine receptor-bearing cells, including neural crest tumor cells, using the sulfhydryl-containing dopamine analogue, 6-mercaptodopamine, in an effort to target and enhance the toxicity of neocarzinostatin for neuroblastoma.

\section{Methods}

Chemicals. Neocarzinostatin was obtained as a sterile solution $(0.5$ $\mathrm{mg} / \mathrm{ml}$ ) in $0.015 \mathrm{M}$ sodium acetate buffer, $\mathrm{pH} 5$, from Dr. William T. Bradner (Bristol-Myers, Wallingford, CT). 6-Mercaptodopamine was synthesized and its identity and purity confirmed by the methods of Chavdarian and Castagnoli (6). $\beta$-Mercaptoethanol, $N$-acetylcysteine, buthionine sulfoximine (BSO), ${ }^{1}$ and dopamine hydrochloride were obtained from Sigma Chemical Co., St. Louis, MO.

Cell culture. $\mathrm{C} 1300$ murine neuroblastoma cells of the subline NB41A3 were obtained from the American Type Culture Collection, Bethesda, MD. Stock cultures were maintained as adherent cultures on plastic with F-10 Nutrient Mixture (Gibco Laboratories, Grand Island, NY) supplemented with $15 \%$ horse serum and $2.5 \%$ FCS. All cultures were incubated at $37^{\circ} \mathrm{C}$. Plating of sister cultures on six-well plates (Gibco) was achieved by harvesting of stock cultures with trypsin. All such plates were incubated overnight before use in these studies.

Adjunctive treatment of NB4IA3 cells with neocarzinostatin and thiols or dopamine for determination of their relative potencies as enhancers of the biological activity of neocarzinostatin. Sister cultures of NB41A3 cells $\left(2 \times 10^{4}\right.$ cells/well) were plated on day -1 . On day 0 , cultures were treated with either neocarzinostatin dissolved in complete medium, or an equal volume of medium. Cells were so treated for $1 \mathrm{~h}$ at $37^{\circ} \mathrm{C}$. All wells were washed twice with serum-free F-10 Nutrient Mixture, and the cells were treated for $4 \mathrm{~h}$ at $37^{\circ} \mathrm{C}$ with one of three thiol reagents (6-mercaptodopamine, $\beta$-mercaptoethanol, or $N$-acetylcysteine) or with dopamine dissolved in serum-free F-10 Nutrient Mixture. All wells were then washed twice with complete medium, and sufficient medium $(4 \mathrm{ml})$ was added to each well to maintain the sister cultures for the duration of the study. The plates were then incubated at $37^{\circ} \mathrm{C}$ for the remainder of the experiment.

Pretreatment with BSO. Where indicated in the text, sister cultures of NB41 A3 cells prepared as noted above were incubated at $37^{\circ} \mathrm{C}$ in the presence of BSO $(1 \mathrm{mM})$ for $24 \mathrm{~h}$. BSO prevents glutathione synthesis and thereby reduces the intracellular sulfhydryl content. Half of the cultures so treated were assayed for sulfhydryl content by the method of Ellman (7) and for glutathione content by the method of Tietze (8). The

1. Abbreviations used in this paper: BSO, buthionine sulfoximine; $\mathrm{NCZ}$, neocarzinostatin; SHDA, sulfhydryl-containing dopamine analogue, 6-mercaptodopamine. 
remaining cultures were treated with neocarzinostatin as detailed above, and incubated at $37^{\circ} \mathrm{C}$ for the remainder of the experiment.

Competitive inhibition by dopamine of the action of 6-mercaptodopamine. Sister cultures of NB41A3 cells were prepared as noted above, and were treated with neocarzinostatin followed by either 6-mercaptodopamine $(52 \mu \mathrm{M})$ alone or 6-mercaptodopamine in the presence of dopamine $(52,130$, or $260 \mu \mathrm{M})$. In all cases, dopamine was added to the cultures before the addition of 6-mercaptodopamine. All experimental cultures and a control sister culture treated identically except for the addition of medium rather than drug in medium were incubated at $37^{\circ} \mathrm{C}$ for $1 \mathrm{~h}$ during the neocarzinostatin phase of the study and for $4 \mathrm{~h}$ during the 6-mercaptodopamine phase of the study. All wells were then washed free of drug and incubated at $37^{\circ} \mathrm{C}$ for the remainder of the experiment.

Determination of cell culture growth and degree of morphologic differentiation. Cell culture growth and degree of morphologic differentiation of the cultures were determined as we have previously described (3). Briefly, cell counts were performed daily by counting the number of cells per high power field. Three fields were counted per well, and the average cell count was determined. The variation in count from field to field was less than $10 \%$ in most cases, and never greater than $30 \%$. Average cell counts for each well on each day were normalized to the average cell count for that well on day 0 . Cells were considered to be "morphologically differentiated" if they fulfilled all of the following criteria: (a) extension of more than two cellular processes; $(b)$ enlargement of the cell body by at least a factor of 2 over control cells (i.e., largest diameter of cell $\geq 50 \mu \mathrm{m}$ ); and $(c)$ at least one prominent nucleolus.

Determination of the relative potencies of thiol reagents as reducing agents for neocarzinostatin in a cell-free system. The assay for thiol potency as a reducing agent for neocarzinostatin in a cell-free system depends on the fact that, when neocarzinostatin is reduced by thiols in the absence of DNA, reduced neocarzinostatin attacks itself, losing biological activity (9). The decreased activity of the drug is assessed, in turn, by testing the ability of the preincubated neocarzinostatin/thiol mixture to alter neuroblastoma cell morphology. The degree of depression of neocarzinostatin activity which results from preincubation with the thiol is, therefore, an indirect measure of the potency of each thiol as a reducing agent for neocarzinostatin.

Neocarzinostatin $(50 \mathrm{nM})$ was incubated alone or in the presence of one of three thiol reagents (6-mercaptodopamine, $\beta$-mercaptoethanol, or $N$-acetylcysteine; $50 \mu \mathrm{M}$ ) in serum-free F-10 Nutrient Mixture (pH $7.5)$ at $37^{\circ} \mathrm{C}$ for $10 \mathrm{~min}$. This solution was then diluted $1: 10$ into the medium overlying each of four sister cultures. The degree of morphologic differentiation of the cultures was then determined as described above (3).

\section{Results}

Effects of adjunctive treatment with neocarzinostatin and 6mercaptodopamine upon murine neuroblastoma cells. We have previously shown that neocarzinostatin is cytostatic for murine neuroblastoma cells, and that it induces neuron-like morphology in these cells in culture (3). Incubation of sister cultures of murine neuroblastoma cells with 6-mercaptodopamine (26$134 \mu \mathrm{M})$ after neocarzinostatin treatment at a dose by itself sufficient to halve the culture growth rate results in a two- to threefold enhancement of the effects of neocarzinostatin upon these cells. Adjunctive treatment enhances both the antimitotic and morphology-altering effects of neocarzinostatin (see Fig. 1, $A$ and $B$ ).

The family of dose-response curves for cell culture growth in the presence of varying doses of neocarzinostatin and 6-mercaptodopamine are bounded at low neocarzinostatin concentration by the control culture growth rate $(100 \%)$, and at high neocarzinostatin concentration by complete suppression of mitosis (cell count no different from that on day 0 ). For this reason, this family of curves splays out maximally at concentrations of neocarzinostatin sufficient to suppress culture growth without completely abolishing it, and the effects of 6mercaptodopamine are most apparent in this range (see Fig. 1, $C$ and $D$ ). At the doses used, 6-mercaptodopamine alone has no effect upon either cell culture growth (data not shown) or cell morphology.

Role of thiols in the effects of neocarzinostatin upon neuroblastoma cells. To determine whether, as would be expected from previous studies $(4,5)$, the sulfhydryl group is the active moiety in enhancement of the action of neocarzinostatin, we tested the effects of sequential treatment with neocarzinostatin and dopamine upon murine neuroblastoma cells. Dopamine $(0-134 \mu \mathrm{M})$ has no effect upon the retardation of cell culture growth or the alteration of cell morphology produced by neocarzinostatin (data not shown).

To conclusively demonstrate that cellular sulfhydryls play a role in the effects of neocarzinostatin upon neuroblastoma cells, we determined the effects of inhibition of glutathione synthesis upon the action of neocarzinostatin. Glutathione is the major source of intracellular sulfhydryl groups. The ratelimiting enzyme for its synthesis can be inhibited by BSO (10). Incubation of NB41 33 cells with $1 \mathrm{mM}$ BSO for $24 \mathrm{~h}$ reduced the intracellular sulfhydryl content from $1.4 \times 10^{-4}$ to $<3.6$ $\times 10^{-5} \mathrm{nmol} /$ cell. Similarly, the intracellular glutathione content dropped from $1.3 \times 10^{-4}$ to $<3.3 \times 10^{-5} \mathrm{nmol} /$ cell with BSO treatment. Fig. 2 shows the effects of this reduction in cellular sulfhydryl content upon cell culture growth suppression $(A)$ and the induction of morphological differentiation $(B)$ by neocarzinostatin. Prior treatment with BSO reduces the effects of neocarzinostatin upon NB41 A3 cells, vis-a-vis both cell culture growth rate and morphological differentiation. Like that of 6-mercaptodopamine, this effect is most pronounced in the "mid-range" of neocarzinostatin concentrations, since it is, by definition, bounded by the growth and differentiation rates of control cultures at one end of the dose range, and by the maximum possible degree of growth rate suppression and differentiation induction at the other. These results demonstrate that, in the absence of added thiols, endogenous thiols play a role in the activation of neocarzinostatin.

Role of the catecholamine moiety in the adjunctive effects of 6-mercaptodopamine and neocarzinostatin. Enhancement of the effects of neocarzinostatin in murine neuroblastoma cells requires the catecholamine moiety, as well as the thiol. At concentrations comparable to the effective concentrations of 6mercaptodopamine, neither $\beta$-mercaptoethanol nor $N$-acetylcysteine, two noncatecholamine thiol compounds, augments the cytostatic or morphologic (see Fig. 3) effects of neocarzinostatin in this system.

If 6-mercaptodopamine is gaining entry into the neuroblastoma cells via their dopamine receptors, then dopamine should be able to compete with 6-mercaptodopamine at the receptor level and, if present in high enough concentration, diminish the effectiveness of 6-mercaptodopamine. Fig. 4 shows that this is indeed the case. Dopamine diminishes the degree of cell culture growth suppression and the frequency of morphologic change obtained with adjunctive treatment. This effect is dose dependent; that is, the degree of competition by dopamine increases with increasing molar excess of dopamine (tested at equimolar concentration and at two- and fourfold molar excess) over the concentration of 6-mercaptodopamine. 


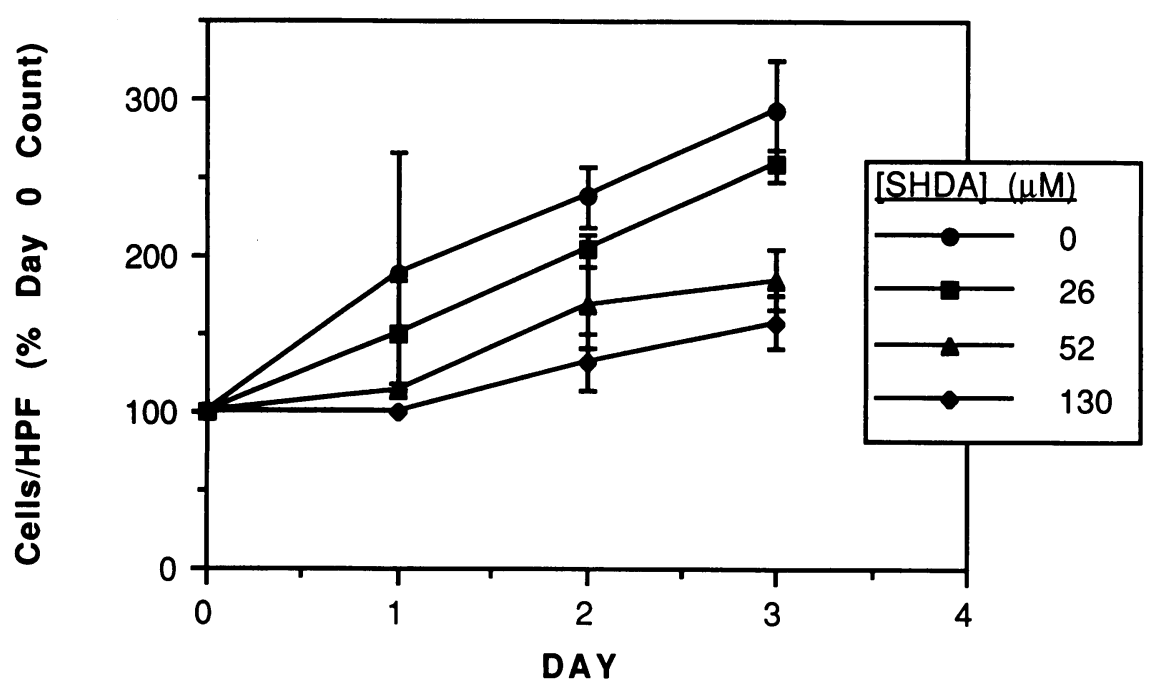

B

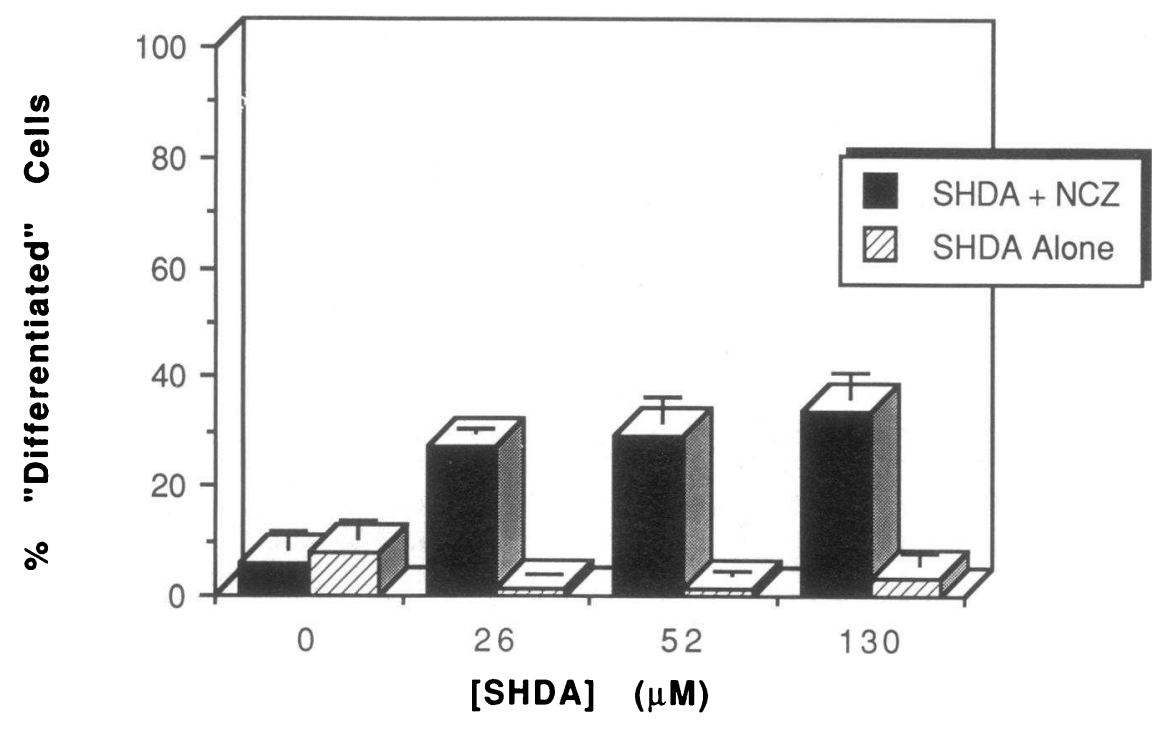

C

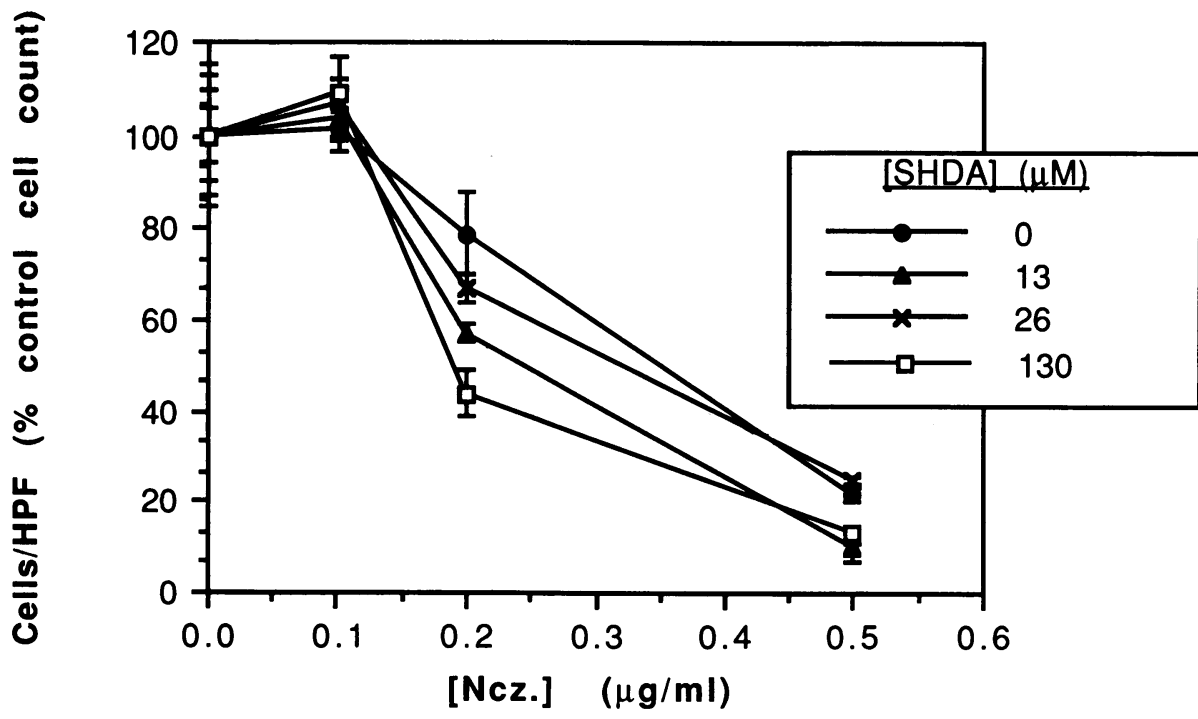

Figure 1. Effects of the adjunctive use of sulfhydryl-containing dopamine analogue, 6-mercaptoethanol (SHDA) and neocarzinostatin (NCZ) upon murine neuroblastoma cell culture growth $(A, C)$ and cell morphology $(B, D) .(A)$ and $(B)$ : Sister cultures were plated on day -1 , and on day 0 , were treated with either $\mathrm{NCZ}$ at a dose sufficient to halve cell culture growth $(A, B)$ or an equal volume of medium $(B)$, followed in each case by treatment with SHDA. NCZ treatment involved incubation with the drug in complete medium (Nutrient Mixture F-10, 15\% horse serum, 2.5\% FCS) for $1 \mathrm{~h}$ at $37^{\circ}$. SHDA treatment involved incubation with the drug in serum-free Nutrient Mixture F-10 for $4 \mathrm{~h}$ at $37^{\circ}$. The methods for cell counting and determination of the percent differentiation of the cultures have previously been described by us (3). All points for this and subsequent figures are plotted as mean ( $n$

$=3$ ) \pm SEM. For panel A, the value on day 3 for $52 \mu \mathrm{M}$ SHDA differs from that for $0 \mu \mathrm{M}$ SHDA with a $P<0.02$ (one-tailed Student's $t$ test). That for $130 \mu \mathrm{M}$ SHDA differs from control with a $P<0.01$. For panel B, the values for 26,52 , and $130 \mu \mathrm{M}$ SHDA $+\mathrm{NCZ}$ differ from that for $0 \mu \mathrm{M}$ $\mathrm{SHDA}+\mathrm{NCZ}$ with a $P<0.002$. Addition of SHDA at these concentrations resulted in a fivefold increase in the percent of cells with the differentiated morphology. $(C)$ and $(D)$ : Sister cultures were plated on day -1 , and on day 0 , were treated with varying concentrations of NCZ followed by SHDA at the concentrations indicated. Experimental conditions were those detailed above. Cell counts and percent differentiation were determined on day 3. Enhancement by SHDA of the antimitotic effects of $\mathrm{NCZ}$ were seen at mid-range concentrations of NCZ, as detailed in the text $(C)$. At all concentrations, SHDA enhanced NCZ-induced differentiation $(D)$ 
D

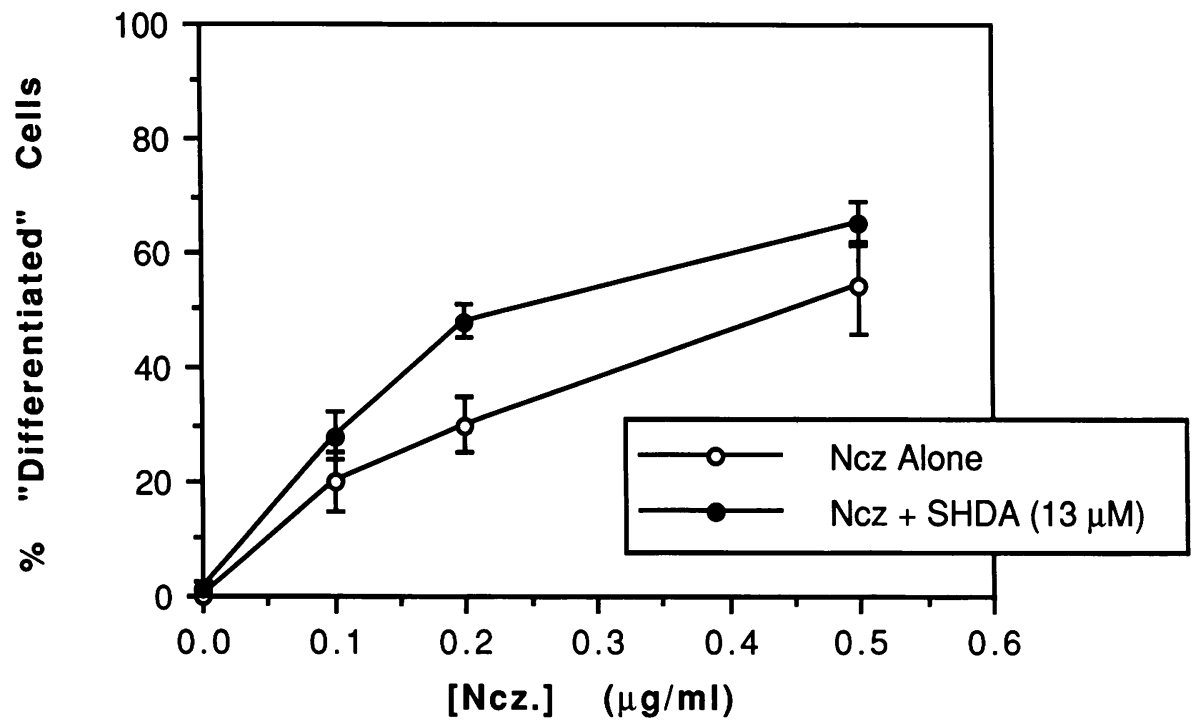

Figure 1 (Continued)

A

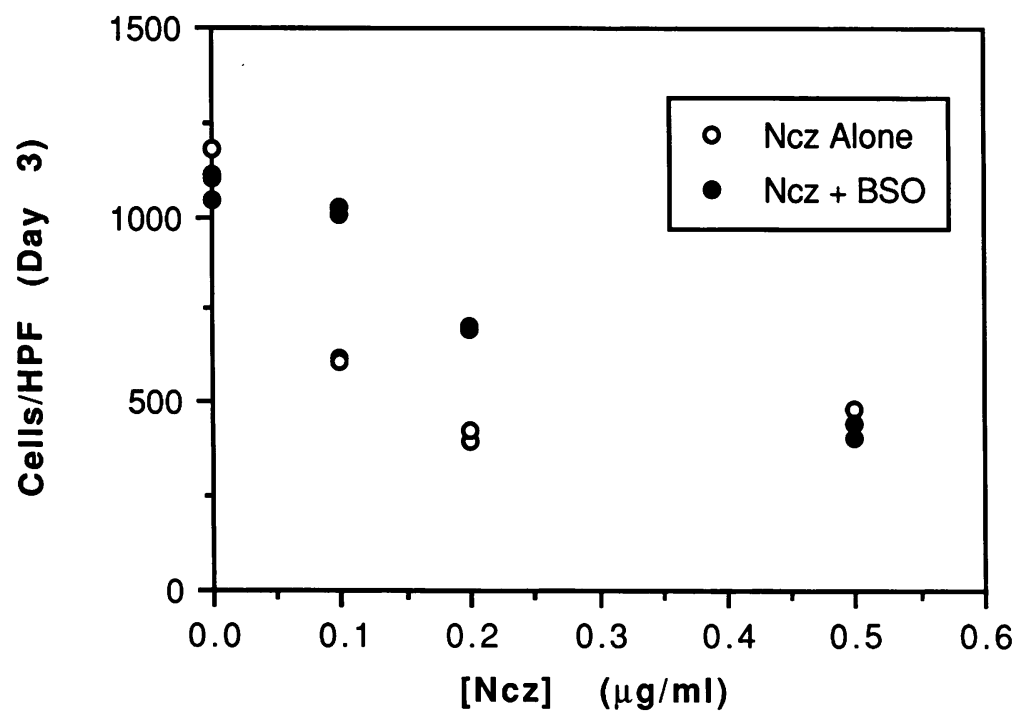

B

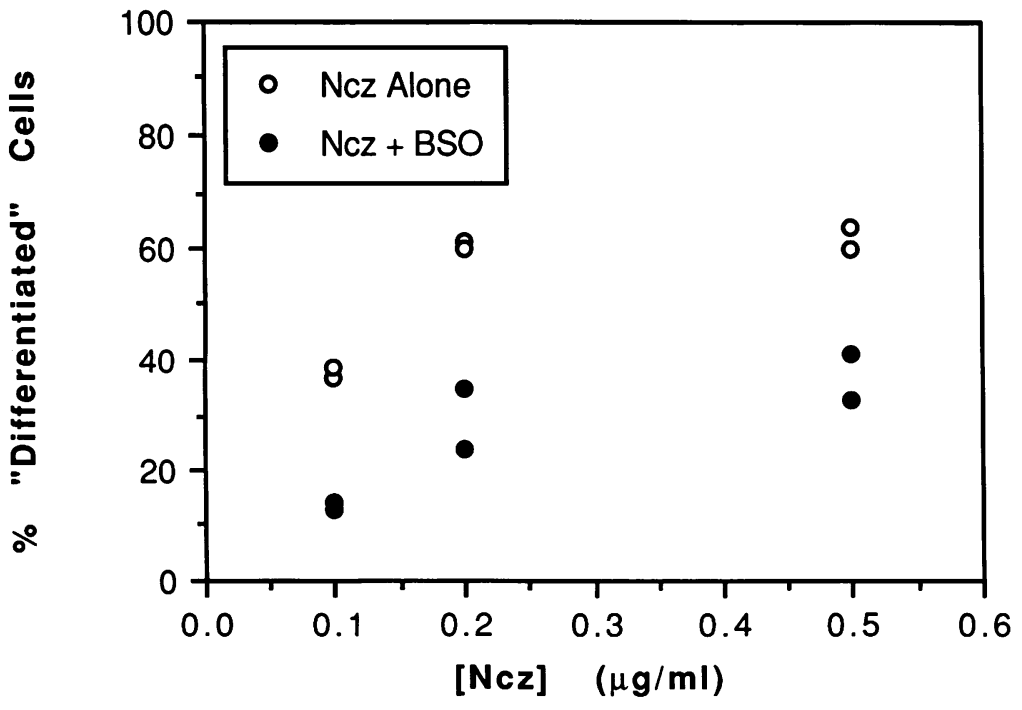

Figure 2. Effects of pretreatment with BSO (1 mM; 24 $\mathrm{h}$ at $37^{\circ}$ ) upon cell culture growth suppression $(A)$ and morphological differentiation $(B)$ by neocarzinostatin. The cell counts and percent differentiation for each of two high power fields on day 3 are shown as a function of NCZ concentration with and without BSO pretreatment. Inhibition of glutathione synthesis by BSO results in diminished suppression of culture growth and decreased induction of morphological differentiation by NCZ. 


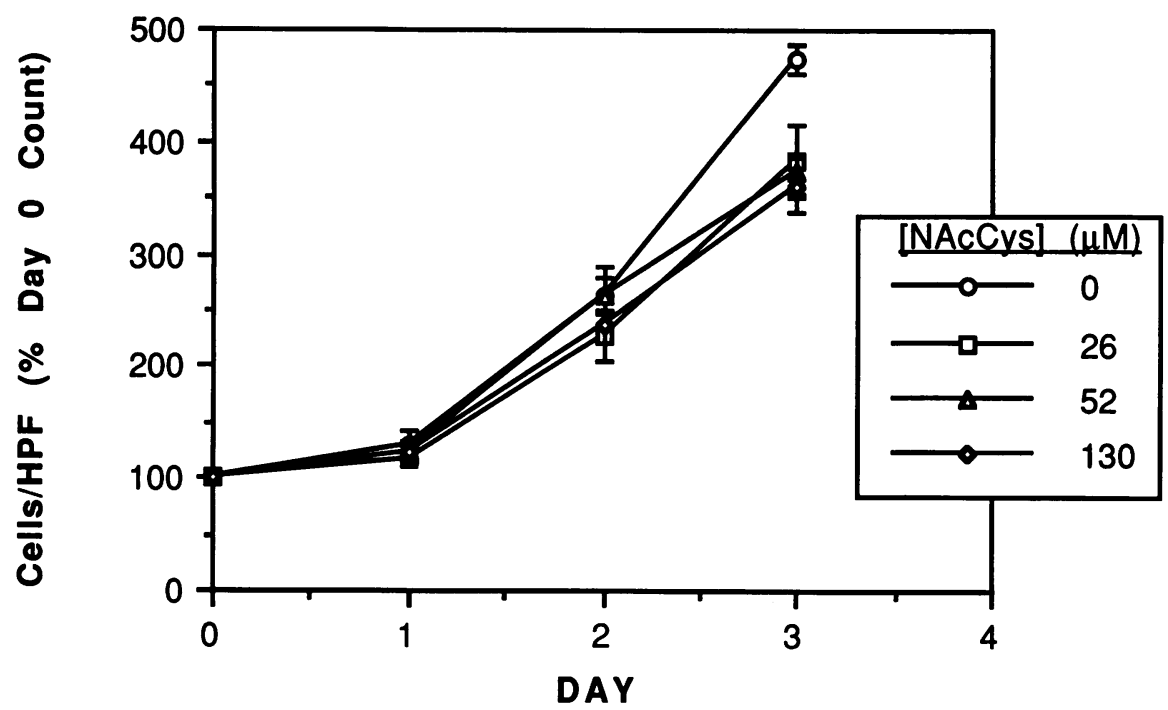

B

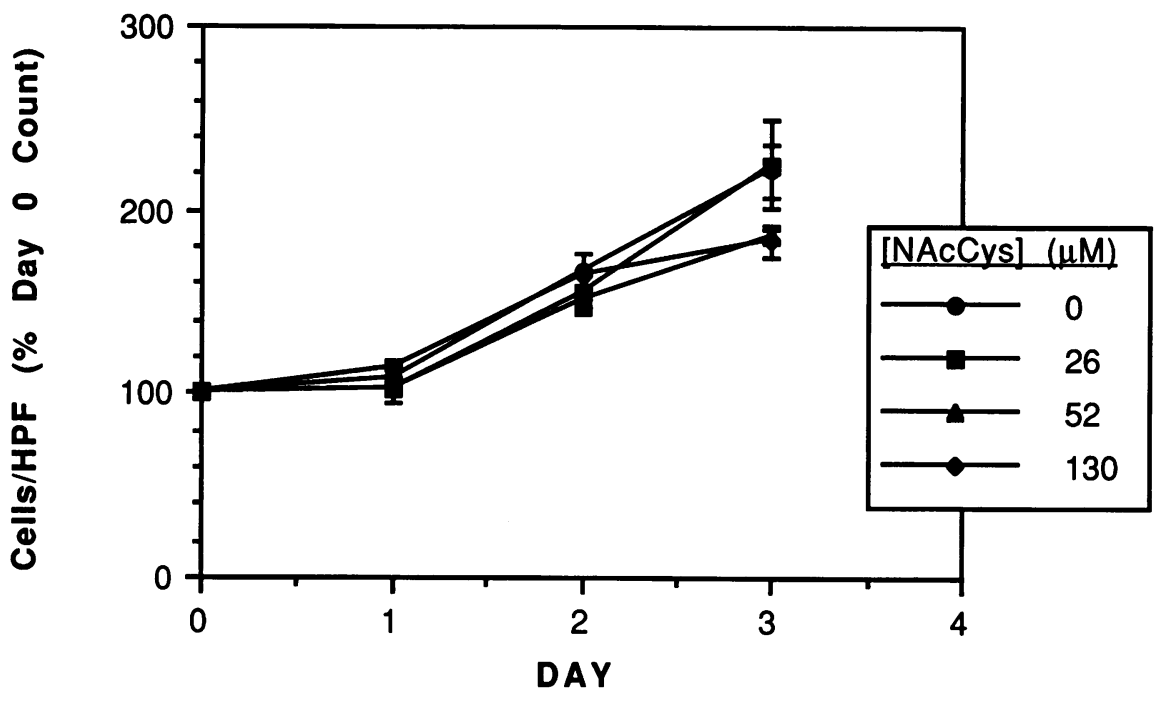

C

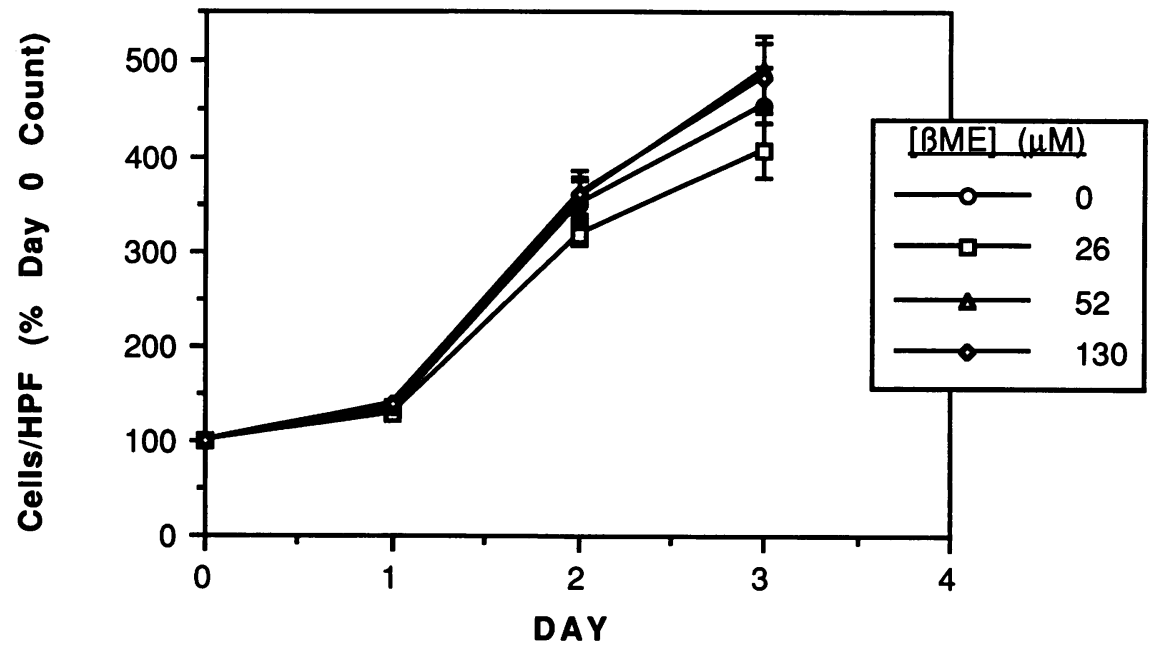

Figure 3. Effects of the adjunctive use of 6-mercaptodopamine (SHDA), $N$-acetylcysteine (NAcCys), or $\beta$-mercaptoethanol $(\beta \mathrm{ME})$, and $\mathrm{NCZ}$ upon the cell culture growth $(A-D)$ and morphology $(E)$ of murine neuroblastoma cells. Culture and treatment conditions were identical to those given in the legend for Fig. 1, except that either SHDA, NAcCys, or $\beta$ ME was used adjunctively with NCZ. Panels A and $B$ show the effects of NAcCys alone $(A)$ and in the presence of NCZ (B). Panels C and D show the effects of $\beta \mathrm{ME}$ alone $(C)$ and in the presence of $\mathrm{NCZ}(D)$. For panel E, "synergistic 'differentiation' " is defined as the response to NCZ plus thiol minus the sum of the response to $\mathrm{NCZ}$ alone and the response to thiol alone. The values for 26,52 , and $130 \mu \mathrm{M}$ SHDA differ from those for $0 \mu \mathrm{M}$ SHDA and those for NAcCys and $\beta \mathrm{ME}$ at all concentrations with a $P$ maximally 0.002 . 
D

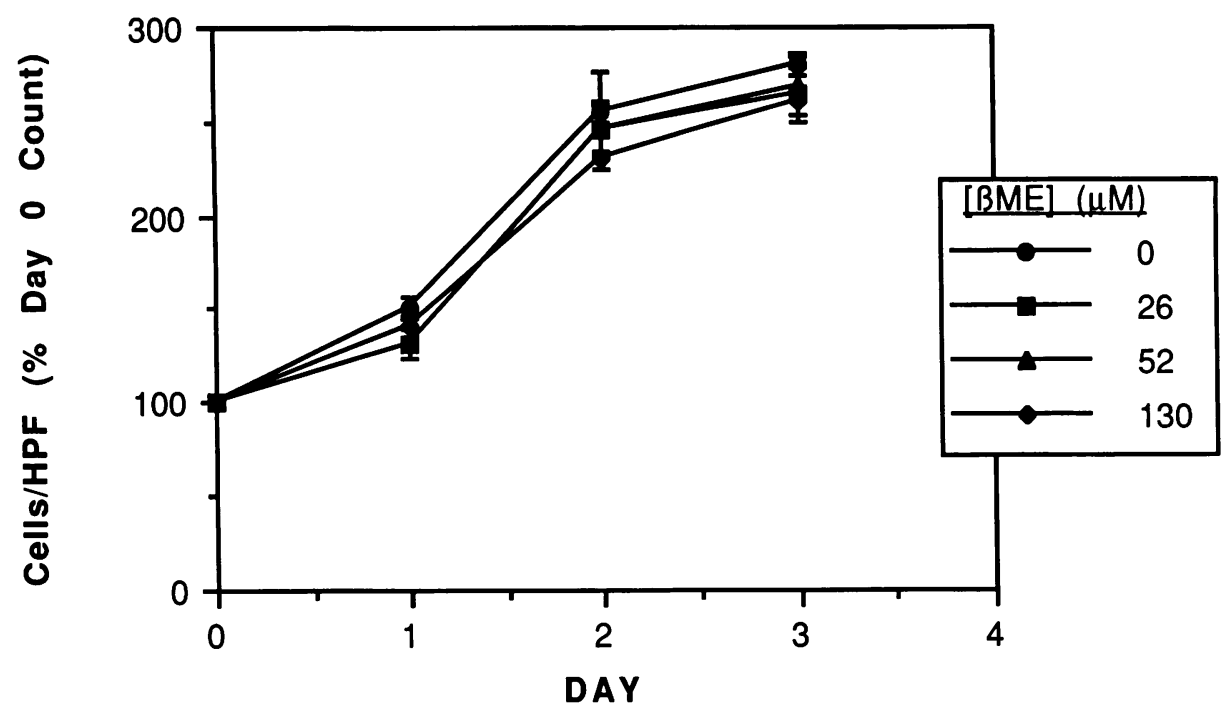

$\mathbf{E}$

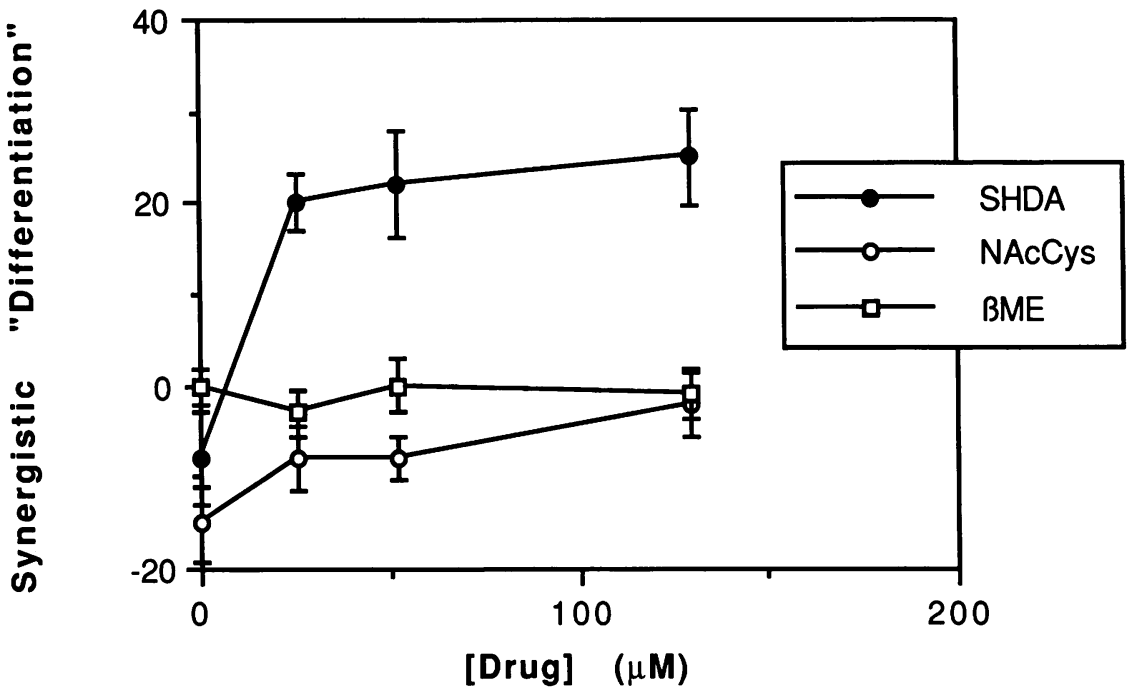

Figure 3 (Continued)

Relative potencies of thiol reagents as reducing agents for neocarzinostatin in a cell-free system. To determine whether the synergy between 6-mercaptodopamine and neocarzinostatin, and the lack of synergy with other thiol agents, is merely the result of a greater ability of the catecholamine mercaptan to chemically reduce neocarzinostatin, we tested the reducing ability of the three thiol compounds in a cell-free system. For this experiment, we used the degree of depression of neocarzinostatin activity which results from preincubation with each thiol as an indirect measure of the potency of that thiol as a reducing agent for neocarzinostatin. As Table I shows, preincubation of neocarzinostatin with either $\beta$-mercaptoethanol, $N$-acetylcysteine, or 6-mercaptodopamine gives comparable degrees of inactivation of neocarzinostatin, as judged by the decreased ability of the preincubated solution to induce morphological change in neuroblastoma cells.

\section{Discussion}

The studies described herein demonstrate that the dose-response curve of murine neuroblastoma cells to the antineoplas- tic agent, neocarzinostatin, can be shifted in the direction of increased potency by the use of the thiol-containing catecholamine, 6-mercaptodopamine. Despite potency equal to that of 6-mercaptodopamine as reductants of neocarzinostatin in cellfree systems, other noncatecholamine thiols are not effective as enhancers of the biological activity of neocarzinostatin in neuroblastoma cells. It is most likely that specific uptake and concentration of the catecholamine mercaptan is responsible for its greater pharmacological activity relative to the other thiol compounds, especially since dopamine competes with 6-mercaptodopamine in this paradigm. That the catecholamine structure alone is not sufficient to enhance the biological activity of neocarzinostatin is shown by the lack of enhancement with dopamine as an adjunctive agent.

Neocarzinostatin has been used in phase I and II studies in adults and has shown some activity against tumors of the urinary tract and prostate $(11,12)$. The discrepancy between its broader spectrum of activity and greater efficacy in vitro than in vivo is thought to be due to problems with penetration of the drug into tissues and with its relatively short half-life in vivo. In addition, its generation of anaphylactic responses in several 


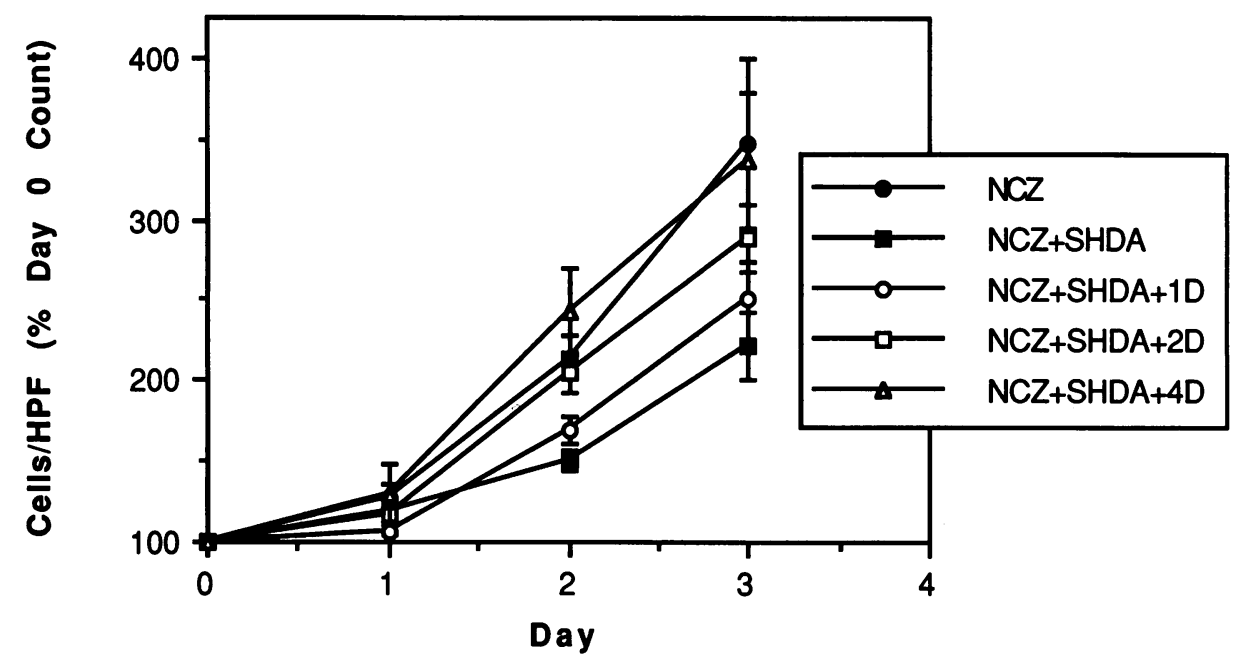

B
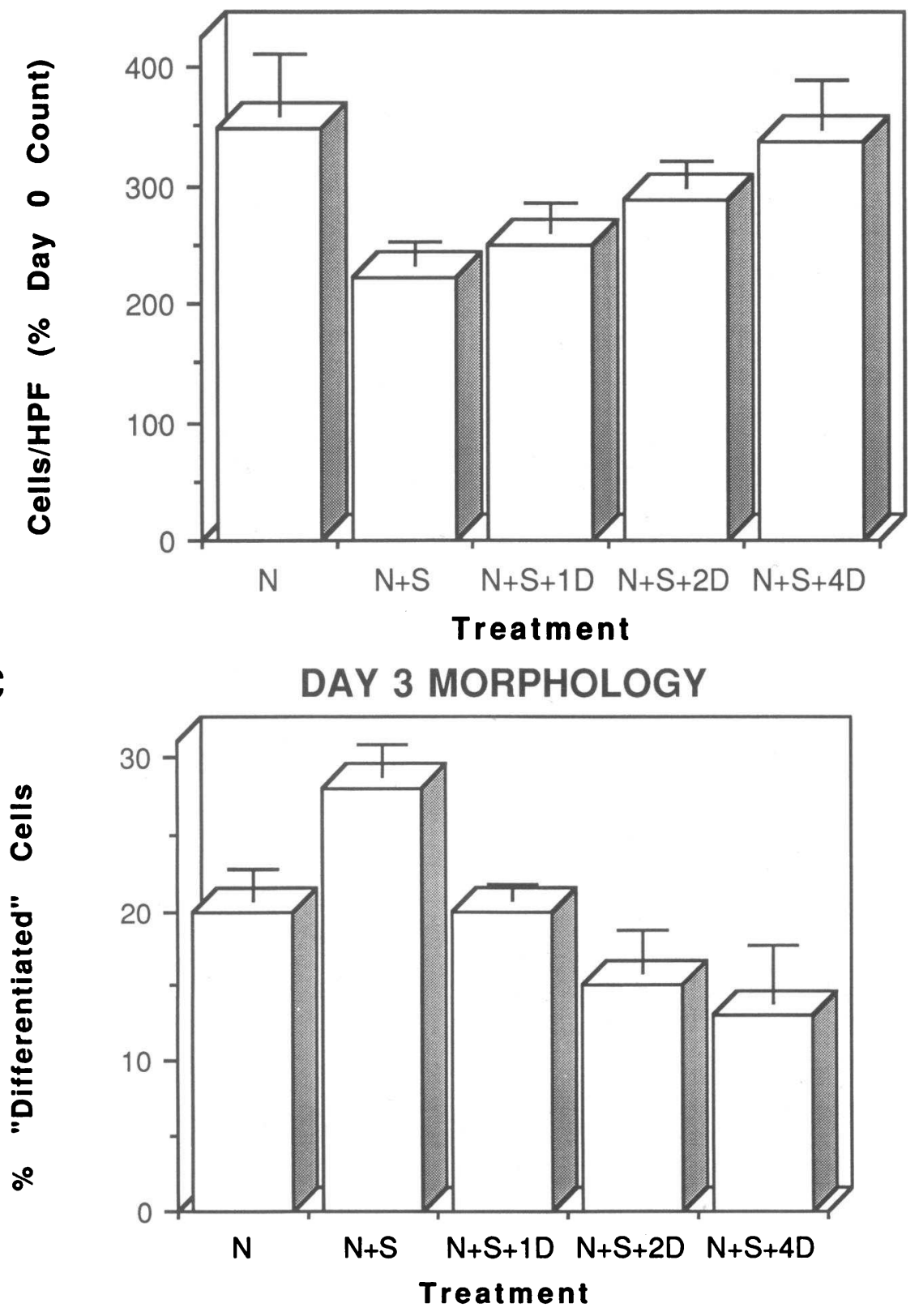
Table I. Effects of Preincubation with SHDA, NAcCys, or $\beta M E$ upon the Biological Activity of NCZ

\begin{tabular}{lc}
\hline $\begin{array}{c}\text { Substance preincubated with } \\
\text { neocarzinostatin }\end{array}$ & $\begin{array}{c}\text { Percent differentiated } \\
\text { cells } \pm \text { SEM }\end{array}$ \\
\hline None & $23 \pm 1$ \\
6-Mercaptodopamine & $12 \pm 3$ \\
N-Acetylcysteine & $10 \pm 0$ \\
$\beta$-Mercaptoethanol & $10 \pm 1$ \\
\hline
\end{tabular}

$\mathrm{NCZ}(50 \mathrm{nM})$ was incubated alone or in the presence of SHDA, NAcCys, or $\beta \mathrm{ME}(50 \mu \mathrm{M})$ in serum-free Nutrient Mixture F-10 $(\mathrm{pH}$ 7.5 ) at $37^{\circ} \mathrm{C}$ for $10 \mathrm{~min}$. This solution was then diluted $1: 10$ into the medium overlying each of four sister cultures. Percent differentiation of the cells was determined as we have previously described (3).

The value for neocarzinostatin incubated in the absence of added thiol reagent (None) differs from that for incubation with 6-mercaptodopamine with a $P$ maximally 0.02 (one-tailed Student's $t$ test). The values for 6-mercaptodopamine, $N$-acetylcysteine, and $\beta$-mercaptoethanol are not significantly different from one another $(P>0.05)$.

patients treated with multiple doses (13), has led to the curtailment of clinical trials in this country. Recent studies suggest that these problems can be overcome by substitution of synthetic polymers for the protein component of neocarzinostatin $(14,15)$.

Neuroblastoma is the single most common solid tumor of childhood. Children with metastatic disease, treated with the most aggressive combinations of surgery, radiation therapy, and chemotherapy, have only a 5-15\% five-year survival rate. This figure has not changed in the last two decades (16). More recent approaches to neuroblastoma have included autologous bone marrow transplantation subsequent to the "purging" of neuroblastoma cells from the marrow (17). The use of so-called differentiation-inducing agents for this purpose has been hampered by the need for agents such as retinoic acid and dibutyryl cAMP to be constantly present for long periods of time to effect terminal physiological changes in the cells $(18,19)$. Our studies show that neocarzinostatin need only be present for $1 \mathrm{~h}$ to obligate morphological change and subsequent cell death (3). This feature, and the fact that the drug could be washed out completely before reinfusion of marrow, obviating the problem of anaphylaxis to the agent, would make neocarzinostatin a practical alternative to more conventional differentiation-inducers. The adjunctive use of neocarzinostatin and 6-mercaptodopamine would be expected to increase the therapeutic index of neocarzinostatin by augmenting the cytotoxicity of the agent for neuroblastoma cells relative to that of marrow stem cells.

The present paper introduces the novel chemotherapeutic strategy of targeted conversion of a prodrug into an active antimitotic agent. This differs substantially from previous chemotherapeutic approaches, which have used maximally toxic agents, and have depended upon quantitative differences between tumor and normal cells or the use of adjunctive drugs to "protect" or "rescue" normal cells from toxicity. Furthermore, the strategy described herein takes specific advantage of the presence of dopamine receptors on neuroblastoma cells. The potential specificity of this approach is twofold. On the one hand, although normal dopaminergic cells would be expected to take up 6-mercaptodopamine, activated neocarzinostatin, an antimitotic agent, should be minimally toxic to this relatively static cell population. On the other, although neocarzinostatin would ordinarily be toxic to rapidly dividing non- neural cells, these cells would not be expected to take up 6-mercaptodopamine, and the low doses of neocarzinostatin which adjunctive treatment facilitates would not by themselves be expected to be toxic. This approach therefore forms a prototype for the development of new chemotherapeutic strategies which take advantage of the physiological characteristics of both the targeted cell population and the excluded cell population.

\section{Acknowledgments}

Thanks are due to Dr. Barbara Imperiale (Carnegie Mellon University, Pittsburgh, PA), and Drs. Alan Kosikowski and Xi Qiang (University of Pittsburgh, Pittsburgh, PA), for synthesizing 6-mercaptodopamine for use in these studies. The author also wishes to express her thanks to Yvette M. Walker-Cox and Karen D. Milligan for expert technical assistance in performing these studies.

These studies were supported by grants from the American Cancer Society (CH-446) and the National Institutes of Health (CA-47161).

\section{References}

1. Calabresi, P., and B. A. Chabner. 1990. Chemotherapy of neoplastic diseases. In The Pharmacological Basis of Therapeutics. A. G. Gilman, T. W. Rall, A. S. Nies, and P. Taylor, editors. Pergamon Press, NY. 1202.

2. Napier, M. A., B. Holmquist, D. J. Strydom, and I. H. Goldberg. 1979. Neocarzinostatin: spectral characterization and separation of a non-protein chromophore. Biochem. Biophys. Res. Commun. 89:635-642.

3. Schor, N. F. 1989. Neocarzinostatin induces neuronal morphology of mouse neuroblastoma in culture. J. Pharm. Exp. Therap. 249:906-910.

4. Kappen, L. S., M. A. Napier, and I. H. Goldberg. 1980. Roles of chromophore and apo-protein in neocarzinostatin action. Proc. Natl. Acad. Sci. USA. 77:1970-1974.

5. DeGraff, W. G., and J. B. Mitchell. 1985. Glutathione dependence of neocarzinostatin cytotoxicity and mutagenicity in Chinese hamster V-79 cells. Cancer Res. 45:4760-4762.

6. Chavdarian, C. G., and N. Castagnoli, Jr. 1979. Synthesis, redox characteristics, and in vitro norepinephrine uptake inhibiting properties of 2-(2-mercapto-4,5-dihydroxyphenyl)ethylamine (6-mercaptodopamine). J. Med. Chem. 22: $1317-1322$

7. Ellman, G. 1959. Tissue sulfhydryl groups. Arch. Biochem. Biophys. 82:7077.

8. Tietze, F. 1969. Enzymatic method for quantitative determination of nanogram amounts of total and oxidized glutathione. Anal. Biochem. 27:502-522.

9. Kappen, L. S., and I. H. Goldberg. 1978. Activation and inactivation of neocarzinostatin-induced cleavage of DNA. Nucleic Acids Res. 5:2959-2967.

10. Seelig, G.F., and A. Meister. 1985. Glutathione biosynthesis. Methods Enzymol. 113:379-390.

11. Natale, R. B., A. Yagoda, R. C. Watson, and D. E. Stover. 1980. Phase II trial of neocarzinostatin in patients with bladder and prostatic cancer. Cancer. 45:2836-2842.

12. Satake, I., K. Tari, M. Yamamoto, and H. Nishimura. 1985. Neocarzinostatin-induced complete regression of metastatic renal cell carcinoma. J. Urol. 133:87-89.

13. McKelvey, E. M., W. Murphy, A. Zander, and G. P. Bodey. 1981. Neocarzinostatin: report of a phase II clinical trial. Cancer Treat. Rep. 65:699-701.

14. Maeda, H., M. Ueda, T. Morinaga, and T. Matsumoto. 1985. Conjugation of poly(styrene-co-maleic acid) derivatives to the antitumor protein neocarzinostatin: pronounced improvements in pharmacological properties. J. Med. Chem. 28:455-461.

15. Oka, K., Y. Miyamoto, Y. Matsumura, S. Tanaka, T. Oda, F. Suzuki, and H. Maeda. 1990. Enhanced intestinal absorption of a hydrophobic polymer-conjugated protein drug, Smancs, in an oily formulation. Pharm. Res. (NY). 7:852855 .

16. Cornaglia-Ferraris, P., M. Ponzoni, P. Montaldo, G. L. Mariottini, E. Donti, D. Di Martino, and G. P. Tonini. 1990. A new human highly tumorigenic neuroblastoma cell line with undetectable expression of $\mathrm{N}$-myc. Pediatr. Res. 27:1-6.

17. Seeger, R. C., K. K. Matthay, J. G. Villablanca, R. Harris, S. A. Feig, M. Selch, D. Stram, and C. P. Reynolds. 1991. Intensive chemoradiotherapy and autologous bone marrow transplantation (ABMT) for high risk neuroblastoma. Proc. Am. Soc. Clin. Oncol. Annu. Meet. Abstr. 1089:251.

18. Prasad, K. N., and S. Kumar. 1975. Role of cyclic AMP in differentiation of human neuroblastoma cells in culture. Cancer. 36:1338-1343.

19. Seeger, R. C., S. E. Siegel, and N. Sidell. 1982. Neuroblastoma: clinical perspectives, monoclonal antibodies, and retinoic acid. Ann. Intern. Med. 97:873-884. 